\title{
Fatty liver and alteration of the gut microbiome induced by diallyl disulfide
}

\author{
YANHONG YANG $^{1,2^{*}}$, FEI YANG ${ }^{2,3^{*}}$, MIAOLING HUANG ${ }^{4 *}$, HUIJUAN WU ${ }^{2,3^{*}}$, \\ CHANGYUAN YANG ${ }^{2,3}$, XINYUE ZHANG ${ }^{2,3}$, LANXIANG YANG ${ }^{2,3}$, GUIBIN CHEN $^{2}$, SHUQI LI ${ }^{4}$,

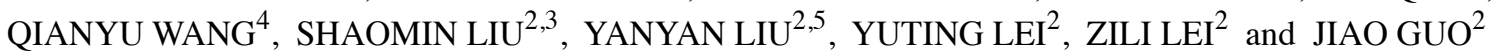 \\ ${ }^{1}$ School of Clinical Medicine, The First Affiliated Hospital, Guangdong Pharmaceutical University, Guangzhou, \\ Guangdong 510080; ${ }^{2}$ Guangdong Metabolic Disease Research Center of Integrated Chinese and Western Medicine, \\ Institute of Chinese Medicinal Sciences, ${ }^{3}$ School of Traditional Chinese Medicine, ${ }^{4}$ School of Pharmacy, \\ ${ }^{5}$ Department of Pathology and Guangdong Key Laboratory for Bioactive Drugs Research, \\ Guangdong Pharmaceutical University, Guangzhou, Guangdong 510006, P.R. China
}

Received April 16, 2019; Accepted August 21, 2019

DOI: $10.3892 / \mathrm{ijmm} .2019 .4350$

\begin{abstract}
Diallyl disulfide (DADS) is one of the primary components of garlic and it exhibits a broad range of biological activities. In the present study, the effects of DADS on lipid metabolism and its potential role in the modulation of the gut microbiome were determined. Hematoxylin and eosin and oil-red $\mathrm{O}$ staining were used to assess the liver and intestinal tissues of mice treated with DADS. The expression of lipid metabolism-associated genes was measured using reverse transcription-quantitative PCR (RT-qPCR). The effects of DADS on the gut microbiome were measured using $16 \mathrm{~S}$ recombinant (r)DNA gene analysis. The results revealed that the serum non-esterified free fatty acids, high density lipoprotein-cholesterol, low density lipoprotein-cholesterol, serum total cholesterol, liver triglyceride and total cholesterol levels of the mice fed with a low-dose of DADS was significantly higher when compared with the control. Hematoxylin and eosin and oil-red $\mathrm{O}$ staining demonstrated that DADS induced fatty liver in mice. The results of the RT-qPCR revealed that the expression levels of a number of lipid metabolism-associated genes were altered in the livers of mice treated with DADS.
\end{abstract}

Correspondence to: Dr Zili Lei or Professor Jiao Guo, Guangdong Metabolic Disease Research Center of Integrated Chinese and Western Medicine, Institute of Chinese Medicinal Sciences, Guangdong Pharmaceutical University, 280 Waihuan East Road, Guangzhou Higher Education Mega Center, Guangzhou, Guangdong 510006, P.R. China

E-mail: 3182683090@qq.com

E-mail: gyguoyz@163.com

${ }^{*}$ Contributed equally

Key words: diallyl disulfide, fatty liver, lipid metabolism, gut microbiome, $16 \mathrm{~S}$ recombinant DNA, gene analysis
The 16S rDNA gene analysis demonstrated that the mice fed on a normal diet treated with a low-dose of DADS had decreased levels of bacteria from the Bacteroidetes phyla and increased levels of bacteria from the Firmicutes phyla. The Kyoto Encyclopedia of Genes and Genomes pathway analysis revealed the top 20 pathways enriched in the low-dose DADS group of mice fed with a normal diet. In the present study, low-dose DADS induced fatty liver and altered the gut microbiota, similar to the phenotype induced by a high fat diet, by regulating the expression of lipid metabolism associated genes.

\section{Introduction}

Garlic (Allium sativum L.) is typically used for culinary purposes (1). Garlic extracts, including diallyl disulfide (DADS), diallyl trisulfide and garlic polysaccharide (GP) and other associated compounds, exhibit a wide range of pharmacological and biochemical activities, including the inhibition of cancer progression, antibacterial and immunomodulatory activity, hypoglycemic effects and antioxidant effects (2-4). As a bioactive component of garlic, DADS exhibits similar biochemical activities. It has been reported that DADS may reverse the epithelial-to-mesenchymal transition in lung cancer and gastric cancer cells $(5,6)$. DADS decreased the proliferation and invasion of osteosarcoma cells by upregulating miR-134 expression (7) and prevented cigarette smoke-induced airway inflammation by inhibiting the phosphorylation of extracellular signal-regulated kinase and the expression of matrix metalloproteinase-9 in lung tissues (8). DADS may also exhibit epigenetic regulation by exerting histone deacetylase-inhibitory activity $(9,10)$.

In recent years, numerous studies have demonstrated an association between altered gut microbiota and a number of diseases, ranging from obesity and inflammatory diseases to behavioral and physiological abnormalities $(11,12)$. Gut microbiota serve an important role in the development of these diseases as it can mediate environmental changes in the intestinal immune system (13). Various environmental factors, 
including diet and medicine, can result in compositional and functional changes to the gut microbiota. Filocamo et al (14) evaluated the effect of garlic powder on the viability of representative gut bacteria in vitro, and the results indicated that garlic intake had the potential to temporarily modulate the gut microbiota. Wang et al (15) reported that GP could be used to protect the liver against fibrosis through modulating lipid peroxidation and oxidative stress, regulating the transforming growth factor- $\beta 1$ and tumor necrosis factor- $\alpha$ signaling pathways, and may be used to treat alcoholic liver fibrosis (ALF) and ALF-induced gut microbiota dysbiosis.

Although it has been reported that DADS has anti-cancer, anti-inflammatory and anti-oxidative effects, its effects on the gut microbiome have not yet been determined. In the present study, C57BL/6 mice were treated with DADS and a high fat diet (HFD), and the effects of DADS on the liver and the intestines were determined. The association between DADS, fatty liver, HFD and gut microbiota was investigated, which may encourage further studies that focus on the roles and underlying molecular mechanisms of DADS on the development of fatty liver.

\section{Materials and methods}

Animals and tissue collection. All experimental procedures in the present study were performed in accordance with the guidelines of the Ethics Committee of Guangdong Pharmaceutical University. The animal experiments were approved by the Committee on Laboratory Animal Care and Use of Guangdong Pharmaceutical University (Guangzhou, China). A total of 60 male C57BL/6 mice (5 weeks of age; weight, $19.92 \pm 1.05 \mathrm{~g}$ ) obtained from the Laboratory Animal Center of Guangdong Province, were housed in a specific pathogen-free mouse facility, at $25^{\circ} \mathrm{C}, 60-65 \%$ humidity, $12 \mathrm{~h}$ light $/ 12 \mathrm{~h}$ dark cycle, with free access to food and water. The mice were randomly divided into 6 groups and each group contained 10 mice. Group 1 (NFD-CON) were fed a control normal food diet (NFD); group 2 (NFD-AL) were fed with a NFD and treated with a low dose of DADS; group 3 (NFD-AH) were fed with a NFD and treated with a high dose of DADS; group 4 (HFD-CON) were fed with a high fat diet (HFD); group 5 (HFD-AL) were fed with a HFD and treated with a low dose of DADS; and group 6 (HFD-AH) were fed with a HFD and treated with a high dose of DADS. The concentration of DADS used in the low-dose and high-dose treated mice were 10 and $20 \mathrm{mg} / \mathrm{kg}$ (weight), respectively. The mice of groups 2 , 3,5 and 6 were treated with DADS by intragastric administration once every day, and were sacrificed after 8 weeks. The human equivalent doses (HED) were calculated by multiplying the mouse doses by 0.081 as previously described (16). HED were 0.81 and $1.62 \mathrm{mg} / \mathrm{kg}$ (weight) respectively, and these doses could be achieved through a regular diet. The dosage of DADS was calculated based on the most recently recorded body weight of the mice, as previously described $(8,17)$.

$16 S$ recombinant (r)DNA gene analysis. Fecal bacterial DNA extraction, the 16S rDNA gene PCR amplification and sequencing, and the $16 \mathrm{~S}$ rDNA gene analysis were performed by Gene Denovo Biotechnology Company (Guangzhou, China). The experimental procedures were performed as previously described (18). The sequencing data were analyzed using Quantitative Insights into Microbial Ecology (QIIME; version 1.9.1; http://qiime.org) (19). The 16S rDNA gene sequences were assigned to operational taxonomic units (OTUs) using Uparse (version 9.2.64_i86linux32; http://www. drive 5.com/uparse) (20) with a threshold of $97 \%$ pair-wise identity, and classified taxonomically using the Ribosomal Database Project classifier (version 2.2; http://rdp.cme.msu. edu/classifier/classifier.jsp) (21). The abundance of bacteria was calculated using the method of Ace and Chao (22), and the diversity of bacteria was estimated using the method of Shannon and Simpson (23). Principal coordinates analysis (Vegan; version 2.5-4; https://cran.r-project.org/web/packages/vegan) was performed in order to present the differences between the gut microbial communities of different groups. The Kyoto Encyclopedia of Genes and Genomes (KEGG) pathway analysis was performed http://www.kegg.jp/kegg/kegg1.html. Linear Discriminant Analysis Effect Size (LEFse) was used to identify the specific bacterial taxa of different groups (24).

Assays of the serum lipid profile. The mice were adequately anesthetized with ether for blood collection prior to sacrifice. The anesthesia method was approved by the Ethics Committee of Guangdong Pharmaceutical University, and the pinch reflex was monitored to ensure full anesthesia. After $1 \mathrm{ml}$ blood collection, the mice were sacrificed by cervical dislocation. The concentrations of serum non-esterified free fatty acids (NEFA), high density lipoprotein-cholesterol (HDL-C), low density lipoprotein-cholesterol (LDL-C), total cholesterol (TC), triglyceride (TG), alanine aminotransferase (ALT) and aspartate aminotransferase (AST), together with liver TC and TG levels were measured according to the manufacturer's protocols for each kit. Determination kits for NEFA, TC, TG, ALT and AST were from Nanjing Jiancheng Bioengineering Institute, and determination kits for HDL-C and LDL-C were from Shanghai Rongsheng Biotechnology Co., Ltd. Fasting blood glucose (FBG) was determined by accu-chek (Roche Diagnostics).

Hematoxylin and eosin and oil-red $O$ staining. Mouse liver and intestine tissues were fixed in $4 \%$ paraformaldehyde at $4{ }^{\circ} \mathrm{C}$ overnight for hematoxylin and eosin staining and oil-red $\mathrm{O}$ staining. H\&E staining was performed on $4-\mu \mathrm{m}$-thick sections, stained with hematoxylin for $3 \mathrm{~min}$ and eosin for $20 \mathrm{sec}$ at room temperature. Oil-red $\mathrm{O}$ staining was performed on $7-\mu \mathrm{m}$-thick frozen sections, stained with oil-red $\mathrm{O}$ for $10 \mathrm{~min}$ at room temperature. The images were captured with a PerkinElmer Automated Quantitative Pathology System (PerkinElmer, Inc.).

Reverse transcription-quantitative (RT-qPCR). Total RNA of mice liver was extracted from samples using TRIzol ${ }^{\circledR}$ reagent (Invitrogen; Thermo Fisher Scientific, Inc.), and were subjected to RT using a PrimeScript ${ }^{\mathrm{TM}}$ RT Reagent kit (Takara Bio, Inc.). The RT conditions were: $37^{\circ} \mathrm{C}$ for $15 \mathrm{~min}$ and $85^{\circ} \mathrm{C}$ for $5 \mathrm{sec}$. The sequences of the primers used in the present study are listed in Table SI (Sangon Biotech (Shanghai) Co., Ltd., China). RT-qPCR was performed using a SYBR Premix Ex Taq kit (Takara Bio, Inc.) and a PikoReal PCR system (Thermo Fisher Scientific, Inc.). The thermocycling conditions 
were: $95^{\circ} \mathrm{C}$ for $30 \mathrm{sec}$; followed by 40 cycles of $95^{\circ} \mathrm{C}$ for $5 \mathrm{sec}$, $60^{\circ} \mathrm{C}$ for $20 \mathrm{sec}$ and $65^{\circ} \mathrm{C}$ for $15 \mathrm{sec}$. GAPDH was used as the reference gene.

Western blotting. The livers of the mice were collected, lysed by Radio-Immunoprecipitation Assay lysis buffer (Dalian Meilun Biotechnology Co., Ltd.) and centrifuged at 13,680 x g, at $4^{\circ} \mathrm{C}$ for $30 \mathrm{~min}$, and the supernatant was frozen at $-80^{\circ} \mathrm{C}$. The concentration of the protein was determined using BCA, equal amounts of protein $(30 \mu \mathrm{g})$ were separated via SDS-PAGE (12\% gel) and transferred to a PVDF membrane. The PVDF membrane was blocked with $5 \%$ skimmed milk for $1 \mathrm{~h}$ at room temperature, and incubated overnight with primary antibodies at $4^{\circ} \mathrm{C}$. Subsequently, after incubation at room temperature for $1 \mathrm{~h}$ with horseradish peroxidase-labeled antibody, the signals were visualized using enhanced chemiluminescence reagent. The primary antibodies included: Mouse anti-hepatic lipase (1:1,000; cat. no. sc-21740; Santa Cruz), mouse anti-HNF4 $\alpha$ (1:5,000; cat. no. ab41898; Abcam), rabbit anti-ABCG5 (1:3,000; cat. no. ab87116; Abcam), rabbit anti-CD36 (1:1,000; cat. no. ab133625; Abcam), rabbit anti-FASN (1:1,000; cat. no. 3180; Cell Signaling Technology, Inc.), rabbit anti-HMGCoAR (1:3,000; cat. no. ab174830; Abcam), mouse anti-SCD1 (1:1,000; cat. no. ab19862; Abcam), and mouse anti-GAPDH (1:2,000; cat. no. ab8245; Abcam). Secondary antibodies included HRP-donkey anti-mouse $\operatorname{IgG}(1: 2,000$; cat. no. ab150105; Abcam) and HRP-goat anti-rabbit IgG (1:2,000; cat. no. os0701; Earthox Life Sciences). The Lane 1D software (version 5.1.0.0; SageCreation) was used for quantification of western blotting bands.

Statistical analysis. Statistical differences were determined using SPSS software (version 23.0; IBM Corp). One-way ANOVA with a least significance difference post hoc test for equal variances, or one-way ANOVA with Games-Howell for unequal variances was used to determine the difference between different groups. The data are presented as the mean \pm standard deviation. Each experiment was repeated at least 3 times. $\mathrm{P}<0.05$ was considered to indicate a statistically significant difference.

\section{Results}

Low-dose treatment with DADS results in fat deposition in the liver of mice on a NFD. There was no significant difference in the body weight of the three groups of mice fed with a NFD; however, the body weight of the groups treated with DADS was increased in the HFD groups compared with the control group of HFD (HFD-AL vs. HFD-CON, P $<0.05$; HFD-AH vs. HFD-CON, P<0.01; Fig. 1A). The FBG of the HFD mice treated with a low dose of DADS was elevated compared with the control group of mice fed with a NFD $(\mathrm{P}<0.05$; Fig. 1B). Serum NEFA levels were increased in the low-dose DADS group of mice fed with a NFD and in the HFD groups (HFD-CON and HFD-AL vs. NFD-CON, P<0.01; HFD-AH and NFD-AL vs. NFD-CON, P<0.001; Fig. 1C). Serum TG levels of the HFD groups treated with low and high doses of DADS were also increased (HFD-AL vs. NFD-CON, P $<0.01$; HFD-AH vs. NFD-CON, P $<0.001$; Fig. 1D). Serum TC, HDL-C and LDL-C of the NFD group treated with low-dose DADS and the HFD groups were increased (HFD-CON vs. NFD-CON, $\mathrm{P}<0.001$; HFD-AL vs. NFD-CON, $\mathrm{P}<0.01$; NFD-AL vs. NFD-CON, P<0.05; Fig. 1E. HFD-CON, HFD-AL and HFD-AH vs. NFD-CON, P<0.001; NFD-AL vs. NFD-CON, P<0.05; Fig. 1F. HFD-CON vs. NFD-CON, $\mathrm{P}<0.01$; HFD-AL and HFD-AH vs. NFD-CON, $\mathrm{P}<0.001$; NFD-AL vs. NFD-CON, P=0.068; Fig. 1G). The TG and TC levels of the liver were determined, and the results revealed that the liver TG and TC levels of the NFD group treated with a low-dose of DADS were significantly increased, and in the HFD groups, only the HFD mice treated with a low-dose of DADS exhibited similar results (HFD-AL vs. NFD-CON, $\mathrm{P}<0.01$; NFD-AL vs. NFD-CON, $\mathrm{P}<0.001$; Fig. $1 \mathrm{H}$. HFD-AL vs. NFD-CON, $\mathrm{P}<0.05$; NFD-AL vs. NFD-CON, $\mathrm{P}<0.01$; Fig. 1I). There were no differences in the food intake, serum AST and ALT levels among the 6 groups (Fig. S1A-C).

The hematoxylin and eosin staining results demonstrated fat deposition in the liver of the NFD groups treated with low and high doses of DADS, similar to that in the HFD groups (Fig. 1J). It seemed that DADS could induce fatty liver development in the mice fed with a NFD, and oil-red O staining was used to further visualize this. From the images obtained from the oil-red $\mathrm{O}$ staining, the results were in agreement with the hematoxylin and eosin staining, particularly in the NFD group treated with a low-dose of DADS, which demonstrated notable fat drops in the liver (Fig. 1K). The hematoxylin and eosin staining of different parts of the intestine in the 6 groups exhibited no noticeable differences (Fig. S1D).

Expression patterns oflipid metabolism-associated genes in the liver of mice treated with DADS. The expression levels of lipid metabolism-associated genes were measured using RT-qPCR. Fig. 2A presents the expression levels of lipin 1, hepatic lipase, patatin-like phospholipase domain-containing protein 2 and peroxisome proliferator-activated receptor $\gamma$ coactivator- $1 \alpha$, which are associated with fatty acid oxidation. The expression levels of hepatic lipase were significantly decreased in the HFD groups and in the NFD group treated with a low-dose of DADS $(\mathrm{P}<0.001)$. The expression levels of PNPLA2 were decreased in the HFD groups treated with low and high doses of DADS (HFD-AL vs. NFD-CON, P<0.05; HFD-AH vs. NFD-CON, $\mathrm{P}<0.01)$. Fig. 2B demonstrates that the expression levels of HNF4 $\alpha$ and FXR, which are associated with the conversion of cholesterol to bile acid, and the expression of HNF $4 \alpha$ and FXR, were significantly decreased in the HFD groups and in the NFD group treated with a low-dose of DADS. The increase in the expression levels of CYP7A1 in the HFD-CON and HFD-AH groups were not statistically significant when compared with the NFD-CON group $(\mathrm{P}=0.16$ and $\mathrm{P}=0.118$ respectively, Fig. 2B). Fig. 2C presents the expression of Abcg5, CD36 and LDLR, which are associated with the transport of lipids. The expression of Abcg5 was significantly decreased in the HFD groups and in the NFD group treated with a low-dose of DADS (NFD-AL, HFD-CON and HFD-AH vs. NFD-CON, P<0.05; HFD-AL vs. NFD-CON, P<0.01), and the expression of CD36 was increased in the HFD group treated with a high-dose of DADS $(\mathrm{P}<0.05)$. Fig. 2D presents the expression of ACC $\alpha 1$, ACC $\beta 1$, Fasn, DGAT1, DGAT2, ELOVL5, SREBP-1, SREBP-2, HMGCoAR, PPAR $\gamma$ and SCD1, which are associated with lipid synthesis. The expression levels of ACC $\alpha 1$, DGAT1, SREBP-2 and PPAR $\gamma$ were significantly decreased in the HFD groups 
A

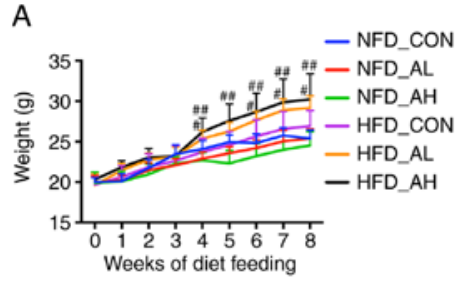

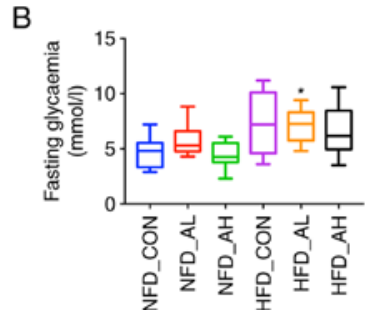

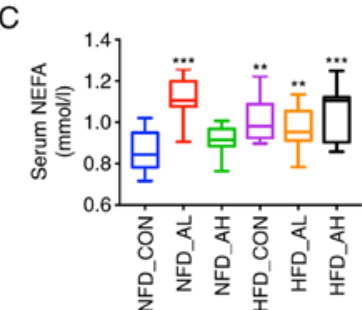

D

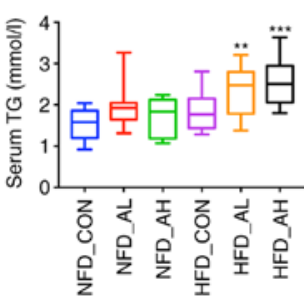

E

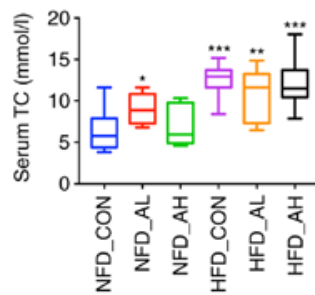

$\mathrm{H}$
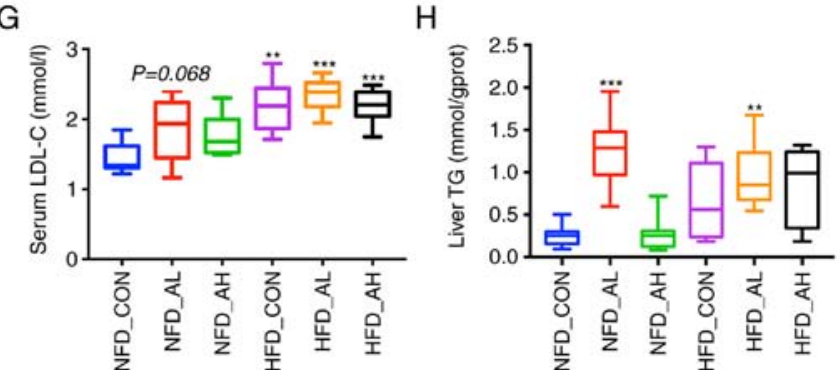

F

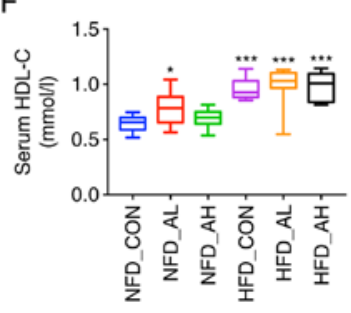

I

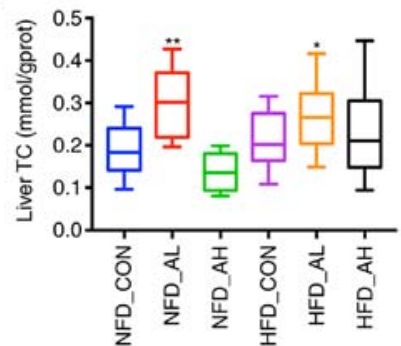

J
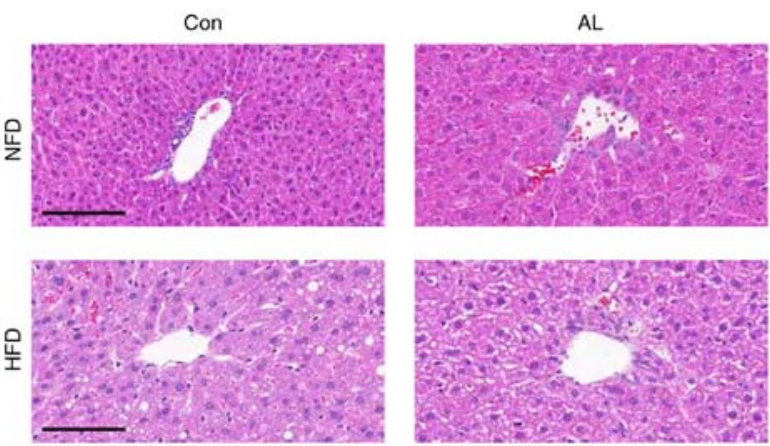

AL
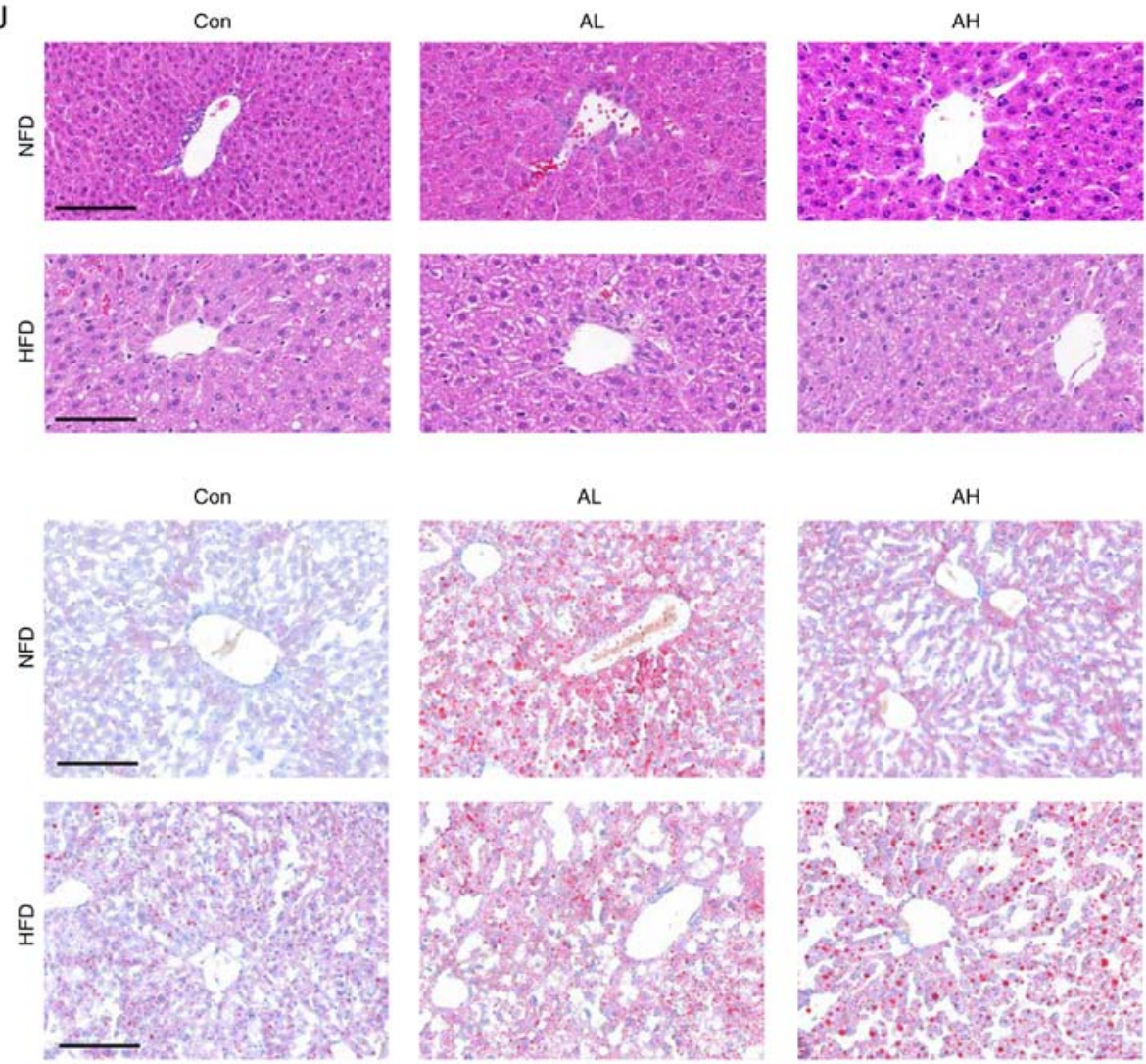

Figure 1. Effect of DADS on lipid metabolism. (A) Effect of DADS and diet groups on the weight of the mice. (B) Effect of DADS on FBG. (C) Effect of DADS on serum NEFA. (D) Effect of DADS on serum TG. (E-G) Effect of DADS on serum TC, HDL-C and LDL-C. (H and I) Effect of DADS on liver TG and TC. (J) Hematoxylin and eosin staining of the liver of the 6 groups of mice. (K) Oil-red $\mathrm{O}$ staining of the 6 groups of mice. Scale bar, $80 \mu \mathrm{m}$. ${ }^{\#} \mathrm{P}<0.05,{ }^{\# \#} \mathrm{P}<0.01$ vs. HFD-CON; ${ }^{*} \mathrm{P}<0.05,{ }^{* *} \mathrm{P}<0.01,{ }^{* * *} \mathrm{P}<0.001$ vs. NFD-CON. NFD-CON, control mice fed with NFD; NFD-AL, mice fed with NFD and treated with a low dose of DADS; NFD-AH, mice fed with NFD and treated with a high dose of DADS; HFD-CON, the control mice fed with HFD; HFD-AL, mice fed with HFD and treated with a low dose of DADS; HFD-AH, mice fed with HFD and treated with a high dose of DADS. NFD, normal food diet; HFD, high fat diet; DADS, diallyl disulfide; NEFA, non-esterified free fatty acids; TG, triglyceride; TC, total cholesterol; HDL-C, high density lipoprotein-cholesterol; LDL-C, low density lipoprotein-cholesterol; FBG, fasting blood glucose. 

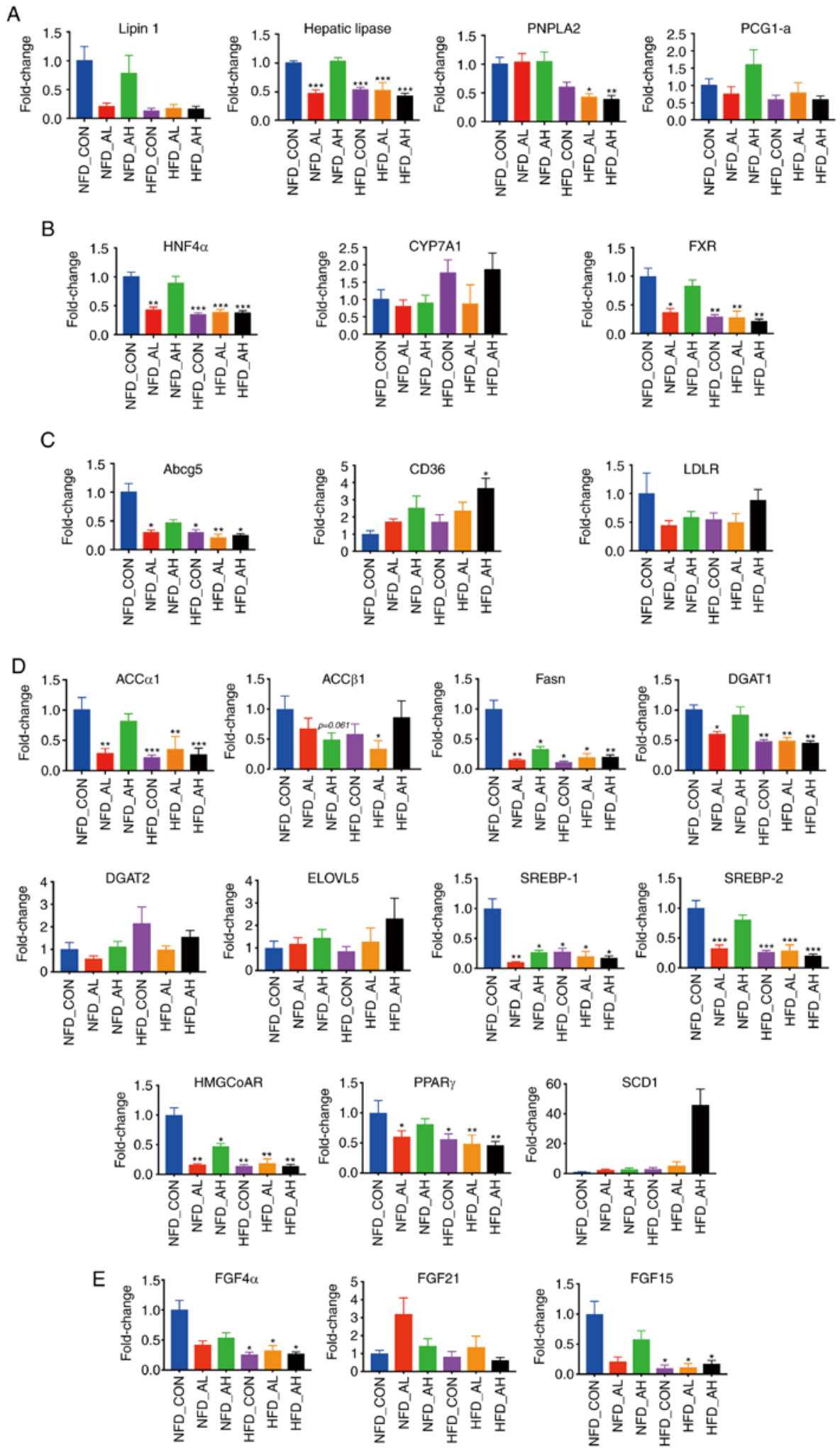

Figure 2. Expression of lipid metabolism-associated genes in the liver of the 6 groups. (A) mRNA expression levels of lipin 1, hepatic lipase, PNPLA2 and PGC-1 $\alpha$. (B) mRNA expression levels of HNF4 $\alpha$, CYP7A1 and FXR. (C) mRNA expression levels of Abcg5, CD36 and LDLR. (D) mRNA expression levels of ACC $\alpha 1$, ACC $\beta 1$, Fasn, DGAT1, DGAT2, ELOVL5, SREBP-1, SREBP-2, HMGCoAR, PPAR $\gamma$ and SCD1. (E) mRNA expression levels of FGF4 $\alpha$, FGF15 and FGF21. "P<0.05, ${ }^{* *} \mathrm{P}<0.01,{ }^{* * *} \mathrm{P}<0.001$ vs. NFD-CON. NFD-CON, control mice fed with NFD; NFD-AL, mice fed with NFD and treated with a low dose of DADS; NFD-AH, mice fed with NFD and treated with a high dose of DADS; HFD-CON, control mice fed with HFD; HFD-AL, mice fed with HFD and treated with a low dose of DADS; HFD-AH, mice fed with HFD and treated with a high dose of DADS. NFD, normal food diet; HFD, high fat diet; DADS, diallyl disulfide; PNPLA2, patatin-like phospholipase domain-containing protein 2; PGC-1 $\alpha$, peroxisome proliferator-activated receptor $\gamma$ coactivator-1 $\alpha$; HNF4 $\alpha$, hepatocyte nuclear factor 4 $\alpha$; CYP7A1, cholesterol 7- alpha hydroxylase gene; FXR, farnesoid X receptor; Abcg5, adenosine triphosphate-binding cassette subfamily G members 5; LDLR, low density lipoprotein receptor; ACC $\alpha 1$, acetyl-CoA carboxylase $\alpha 1$; ACC $\beta 1$, acetyl-CoA carboxylase $\beta 1$; Fasn, fatty acid synthase; DGAT1, diacylglycerol acyltransferase 1; DGAT2, diacylglycerol acyltransferase 2; ELOVL5, elongases of very long-chain fatty acids 5; SREBP-1, sterol regulatory element-binding protein-1; SREBP-2, sterol regulatory element-binding protein-2; HMGCoAR, hydroxy-methyl-glutaryl coenzyme A reductase; PPAR $\gamma$, peroxisome proliferator-activated receptor $\gamma$; SCD1, Stearoyl-CoA desaturase-1; FGF4 $\alpha$, fibroblast growth factor $4 \alpha$; FGF15, fibroblast growth factor 15; FGF21, fibroblast growth factor 21. 

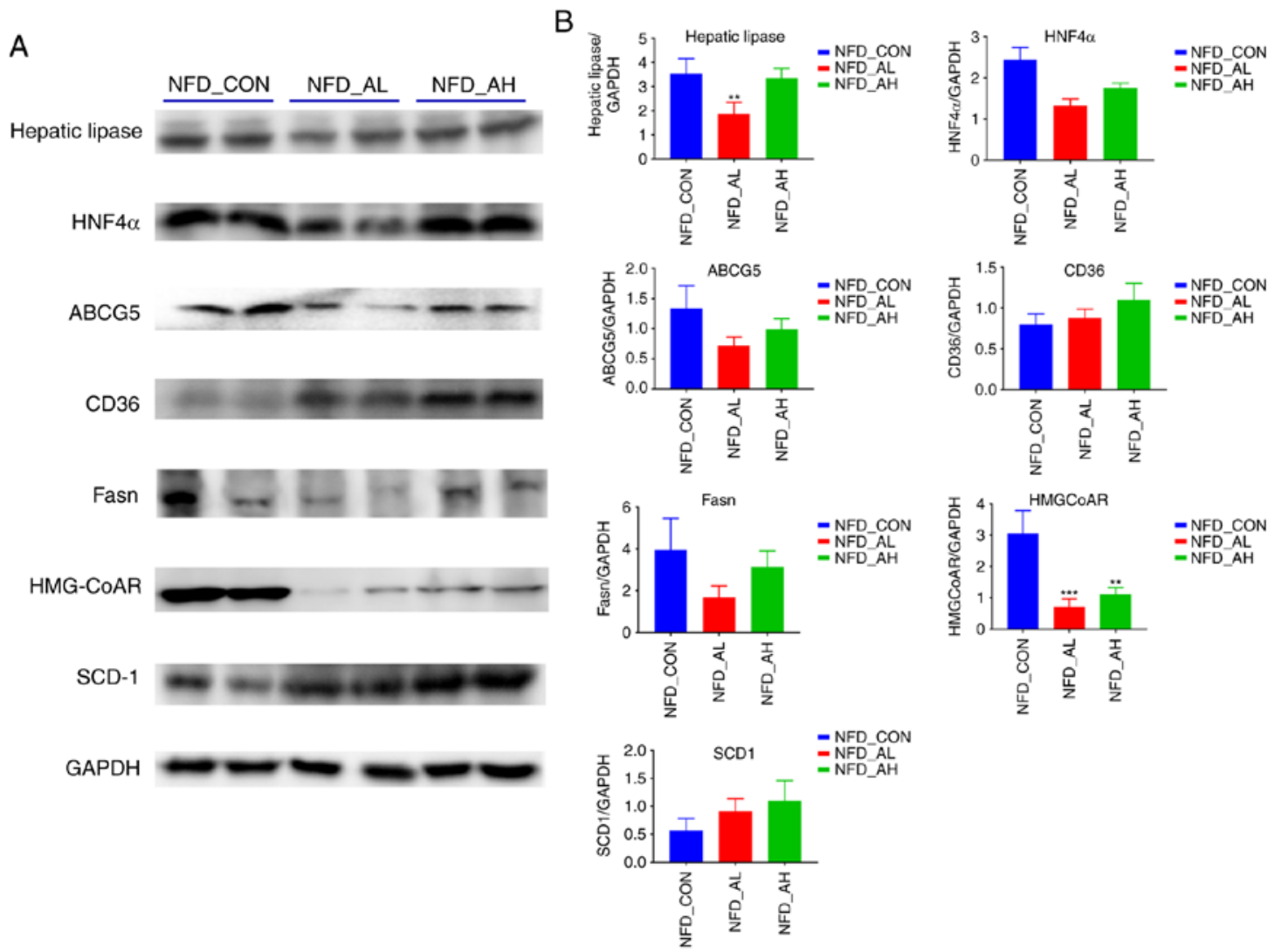

Figure 3. Protein expression of lipid metabolism-associated genes in the liver of the NFD groups. (A) The expression of hepatic lipase, HNF4 $\alpha$, ABCG5, CD36, FASN, HMGCoAR, SCD-1 proteins was detected by western blotting. (B) The relative protein levels of hepatic lipase, HNF4 $\alpha$, ABCG5, CD36, FASN, HMGCoAR and SCD-1. The results are presented as the mean \pm standard deviation. $n=6 .{ }^{* *} \mathrm{P}<0.01,{ }^{* * *} \mathrm{P}<0.001$ vs. NFD-CON. NFD-CON, control fed with NFD; NFD-AL, mice fed with NFD and treated with a low dose of DADS; NFD-AH, mice fed with NFD and treated with a high dose of DADS. NFD, normal food diet; DADS, diallyl disulfide; HNF4 $\alpha$, hepatocyte nuclear factor $4 \alpha$; ABCG5, adenosine triphosphate-binding cassette subfamily G members 5; FASN, fatty acid synthase; HMGCoAR, hydroxy-methyl-glutaryl coenzyme A reductase; SCD1, Stearoyl-CoA desaturase-1.

and in the NFD group treated with a low-dose of DADS (for ACC $\alpha 1$, HFD-CON and HFD-AH vs. NFD-CON, P<0.001; NFD-AL and HFD-AL vs. NFD-CON, P $<0.01$. For DGAT1, HFD-CON, HFD-AL and HFD-AH vs. NFD-CON, P<0.01; NFD-AL vs. NFD-CON, $\mathrm{P}<0.05$. For SREBP-2, $\mathrm{P}<0.001$. For PPAR $\gamma$, HFD-AL and HFD-AH vs. NFD-CON, P<0.01; HFD-CON and NFD-AL vs. NFD-CON, P<0.05), and the expression levels of Fasn, SREBP-1 and HMGCoAR were decreased in the HFD groups and in the NFD groups treated with low and high-doses of DADS compared with the NFD control (for Fasn, NFD-AL and HFD-AH vs. NFD-CON, $\mathrm{P}<0.01$; NFD-AH, HFD-CON and HFD-AL vs. NFD-CON, $\mathrm{P}<0.05$. For SREBP-1, NFD-AL vs. NFD-CON, $\mathrm{P}<0.01$; NFD-AH, HFD-CON, HFD-AL and HFD-AH vs. NFD-CON, $\mathrm{P}<0.05$. For HMGCoAR and NFD-AH vs. NFD-CON, $\mathrm{P}<0.05$; NFD-AL, HFD-CON, HFD-AL and HFD-AH vs. NFD-CON, $\mathrm{P}<0.01)$. Fig. $2 \mathrm{E}$ presents the expression of FGF4 $\alpha$, FGF15 and FGF21, which are associated with insulin resistance (IR). The expression levels of FGF4 $\alpha$ and FGF15 were decreased in the HFD groups $(\mathrm{P}<0.05)$. The expression pattern of lipid metabolism-associated genes in the liver of the NFD groups is presented in Fig. S2.

The protein expression levels of hepatic lipase, HNF4 $\alpha$, ABCG5, CD36, Fasn, HMGCoAR, SCD1 in the NFD groups was determined using western blotting (Fig. 3A-B). The results revealed that the protein expression levels of hepatic lipase was significantly decreased in the NFD group treated with a low-dose of DADS compared with the control and high-dose groups $(\mathrm{P}<0.01$; Fig. 3B); the expression levels of HMGCoAR protein was significantly decreased in the low and high-dose treated groups $(\mathrm{P}<0.001$ for NFD-AL group; $\mathrm{P}<0.01$ for NFD-AH group; Fig. 3B); the expression levels of HNF4 $\alpha$, ABCG5 and Fasn were markedly decreased in the low and high-dose treated groups compared with the control (Fig. 3B); the expression levels of CD36 and SCD1 protein were notably increased in the low and high-dose treated groups (Fig. 3B). The expression at the protein level was concomitant with the mRNA expression levels.

Overview of the $16 S \mathrm{rDNA}$ gene analysis. In order to characterize the gut microbiota of the mice treated with DADS, fecal samples were collected, DNA was purified and amplified, and 16S rRNA gene tags were sequenced. The total tags and operational taxonomic units (OTUs) of each sample are presented in Table SII. The abundance of bacteria was calculated by the method of Ace and Chao (22), and the diversity of bacteria was estimated using the method of Shannon and Simpson (23). All the indices are summarized in Table SII. Fig. 4A demonstrates that the Shannon rarefaction curves for each group reached a saturation plateau, suggesting enough sequence coverage of samples to describe the composition of bacteria. The Venn diagram and the histogram of the six 

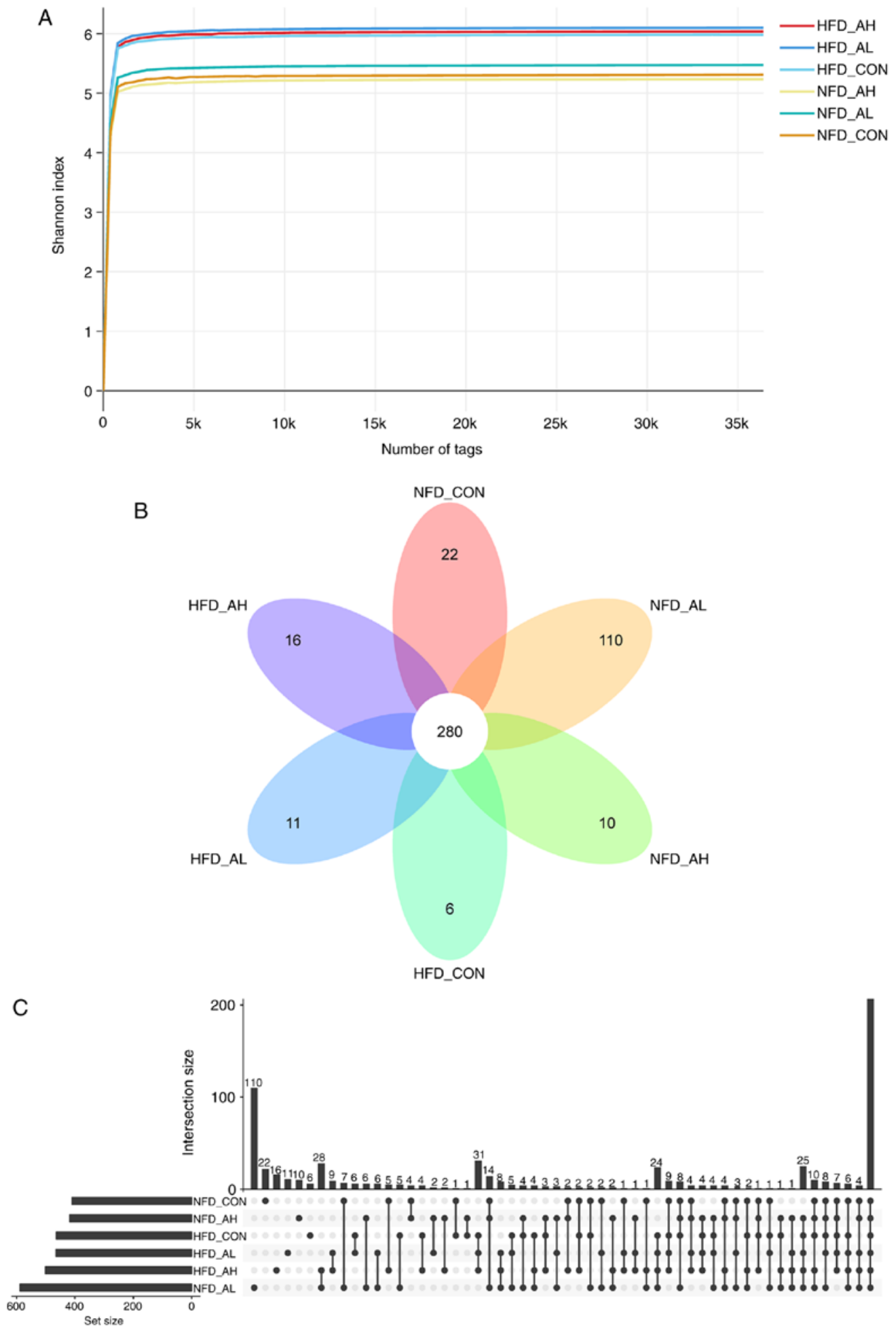

Figure 4. Overview of the 16S rDNA gene analysis. (A) Shannon rarefaction curves for each group. (B and C) Distribution of OTUs in the six groups. NFD-CON, control mice fed with NFD; NFD-AL, mice fed with NFD and treated with a low dose of DADS; NFD-AH, mice fed with NFD and treated with a high dose of DADS; HFD-CON, control mice fed with HFD; HFD-AL, mice fed with HFD and treated with a low dose of DADS; HFD-AH, mice fed with HFD and treated with a high dose of DADS. NFD, normal food diet; HFD, high fat diet; DADS, diallyl disulfide.

different groups demonstrated that there were 280 common OTUs in the six groups of mice (Fig. 4B and C). The Shannon rarefaction curves and the Venn diagram of the NFD groups and the HFD groups are presented in Fig. S3.

Effect of DADS on the relative composition and diversity of gut microbial communities of mice. All sequences were classified from phylum to species, and the three NFD groups exhibited notably different taxonomic compositions. Fig. 5A presents the Unifrac analyses of the three groups of mice fed with a NFD, and the results revealed notable differences among the three groups. A PCoA was also performed, and the results revealed that the control, low-dose DADS and high-dose groups could be distinguished (Fig. 5B), indicating 
A
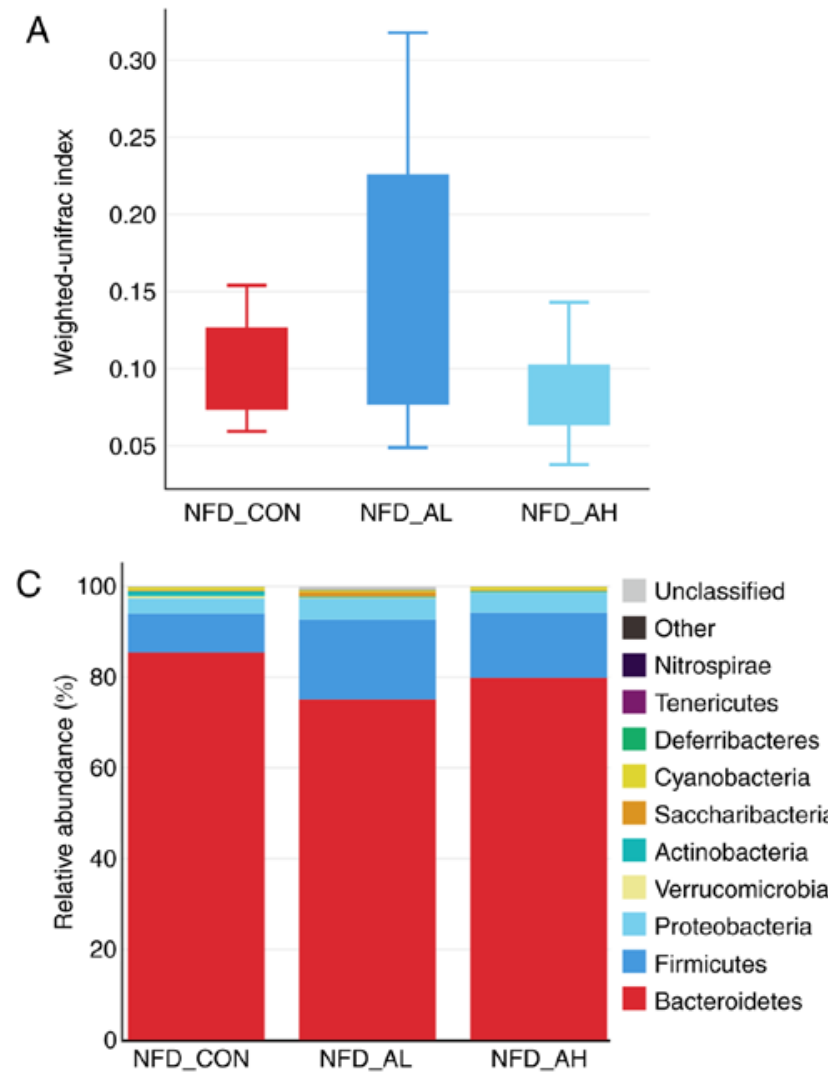

B

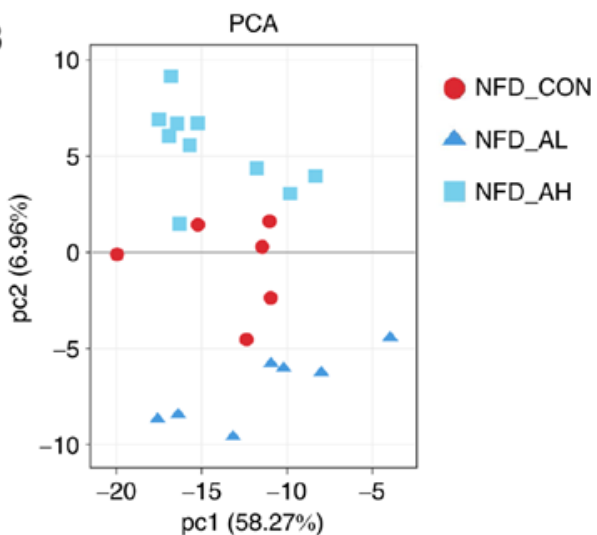

D

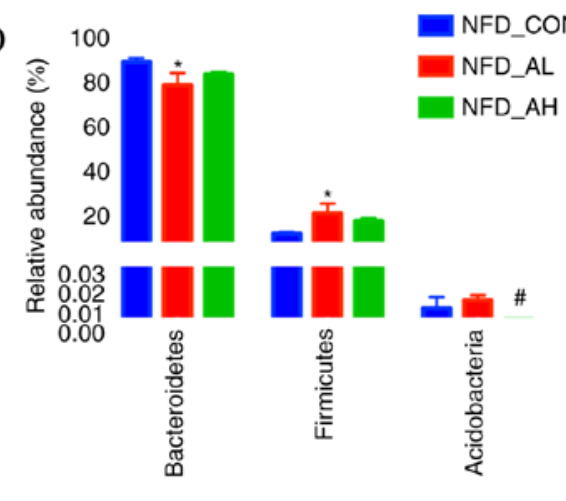

Figure 5. Unifrac analyses, relative composition and heat map of the dominant bacterial family of the gut microbiota in the NFD groups. (A and B) Unifrac analyses. (C and D) Relative composition of the gut microbiota at the bacterial phylum level. ${ }^{*} \mathrm{P}<0.05$ vs. NFD_CON, ${ }^{\sharp} \mathrm{P}<0.05$ vs. NFD_AL. NFD-CON, control mice fed with NFD; NFD-AL, mice fed with NFD and treated with a low dose of DADS; NFD-AH, mice fed with NFD and treated with a high dose of DADS NFD, normal food diet; DADS, diallyl disulfide.

the differences between the three gut microbial communities, suggesting that DADS altered the bacterial community of the mice, and the alteration depended on the dose received. The Unifrac analyses of the six groups are presented in Fig. S4A and B.

The taxonomic compositions of the bacterial phylum in NFD groups are presented in Fig. 5C. The majority of the samples exhibited high percentages of Bacteroidetes, Firmicutes and Proteobacteria, and the low-dose DADS treated group had decreased Bacteroidetes and increased Firmicutes levels compared with the control and high-dose groups (Fig. 5C and D). The taxonomic compositions of the six groups, including the HFD and NFD groups, on phylum and order level are presented in Fig. S4C-F.

In order to identify the specific bacterial taxa associated with DADS, the gut microbiota in the NFD groups were compared using LEFse analysis. Fig. 6A and B demonstrates the specific and predominant bacteria of the 3 groups, revealing that Coriobacteriaceae-UCG was predominant in the control group; Ruminococcus-flavefac and Erysipelatoclostridium were predominant in the high-dose group; and Nitrospira, Nitrospirales, Nitrospirae, Haliangium, Haliangiaceae and Ruminantium-group were specific to the low-dose group. These different taxa may be used as distinguishing biomarkers. The different bacterial taxa in the HFD groups are presented in Fig. S5A and B.

For the NFD groups, the results of the KEGG pathway analysis demonstrated that the top 20 pathways, including 'purine metabolism', 'pyrimidine metabolism', 'amino sugar and nucleotide sugar metabolism', were significantly upregulated in the mice treated with a low-dose of DADS compared with the control group and the mice treated with a high-dose of DADS (Fig. 6C). The full results of the KEGG pathway analysis of the six groups is presented in Fig. S5C. The top 19 pathways that were significantly upregulated in the HFD groups were: 'Transporters', 'ABC transporters', 'DNA repair and recombination proteins', 'Ribosome', 'Purine metabolism', 'Peptidases', 'Pyrimidine metabolism', 'Two-component system', 'Chromosome', 'Amino acid-related enzymes', 'Transcription factors', 'Amino sugar and nucleotide sugar metabolism', 'Methane metabolism', 'Ribosome Biogenesis', 'Arginine and proline metabolism', 'DNA replication proteins', 'Aminoacyl-tRNA biosynthesis', 'Carbon fixation pathways in prokaryotes', 'Alanine, aspartate and glutamate metabolism', compared with the NFD groups (Fig. S5C). In Fig. S5C, the top 20 pathways of the NFD group treated with a low-dose of DADS were also upregulated, particularly the 'Oxidative phosphorylation pathway' compared with the NFD control group and the NFD group treated with a high-dose of DADS. The top 19 pathways in the HFD group treated with a low-dose of DADS were not significantly upregulated compared with the HFD control or HFD groups treated with a high-dose of DADS, and the 'Oxidative phosphorylation pathway' was significantly downregulated in the HFD group treated with a low-dose of DADS (Fig. S5C). Fig. S5D and E indicate an association between fat level, liver TG and TC, and the bacterial taxa. 


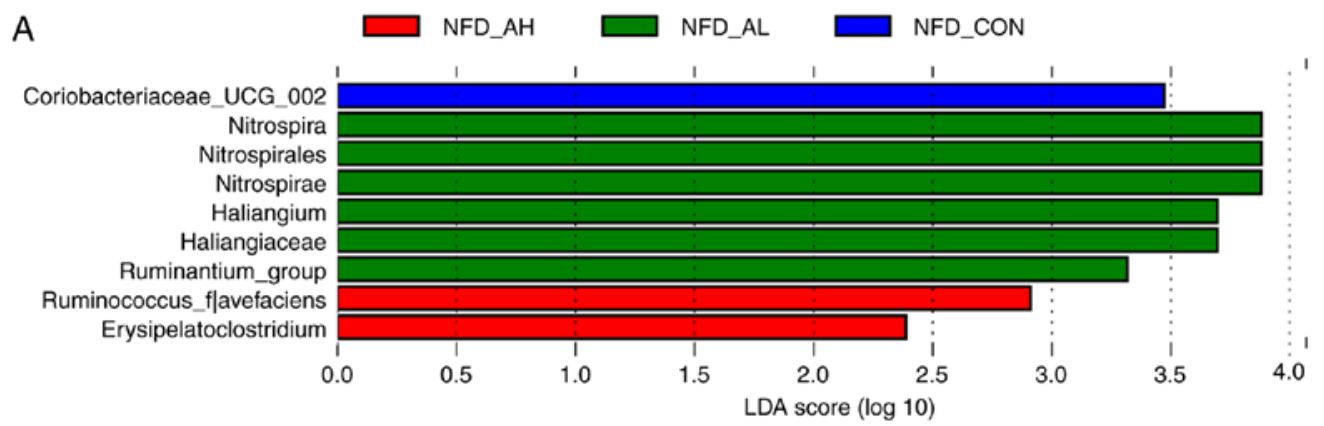

B

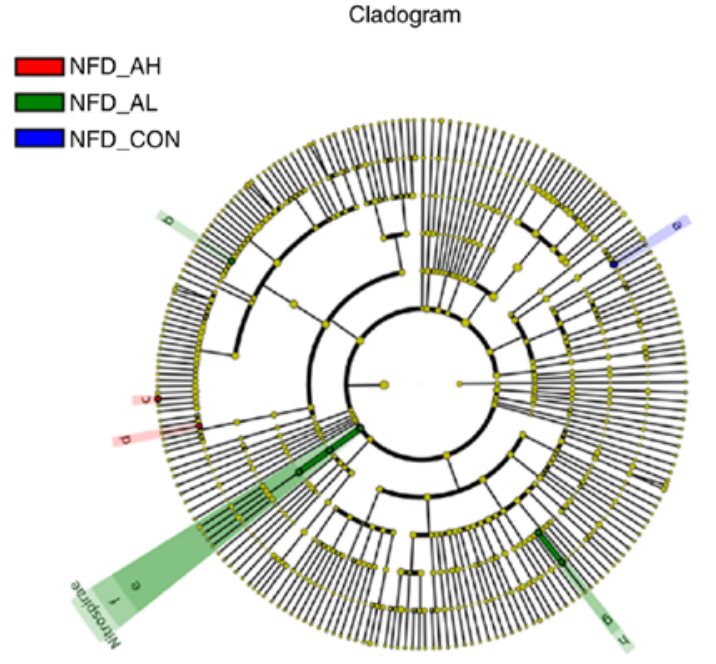

C

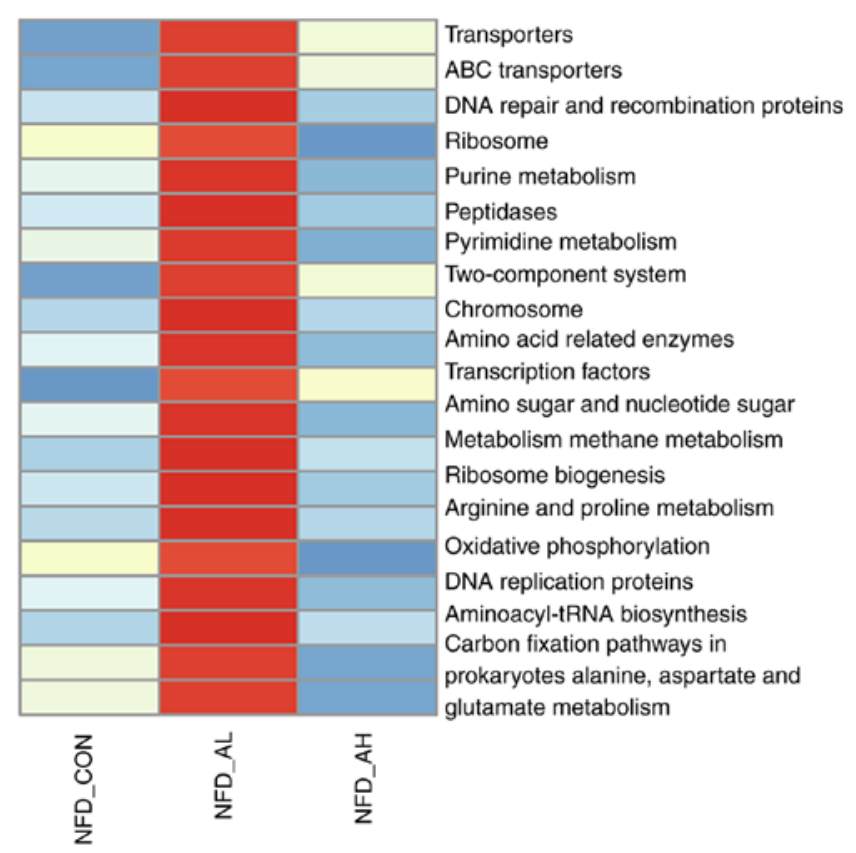

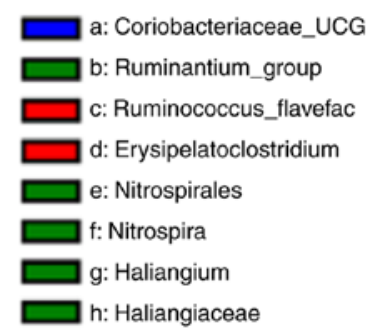

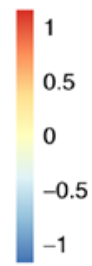

Figure 6. LEFse analysis and KEGG assignments of the NFD groups. (A and B) LEFse analysis identified the predominant taxons of each group. Red represents the bacteria specific for the NFD_AH group, green represents the bacteria specific for the NFD_AL group and blue represents the bacteria specific for the NFD_CON group. (C) Presentation of the altered KEGG pathways in each group. Red represents upregulation, and blue represents downregulation. NFD-CON, control mice fed with NFD; NFD-AL, mice fed with NFD and treated with a low dose of DADS; NFD-AH, mice fed with NFD and treated with a high dose of DADS. NFD, normal food diet; DADS, diallyl disulfide; KEGG, Kyoto Encyclopedia of Genes and Genomes.

\section{Discussion}

Although DADS has been demonstrated to serve a variety of biological and pharmaceutical activities, its effects on the gut microbiota have not yet been determined. In the present study, C57BL/6 mice were treated with DADS and fed with a HFD, and the effects of DADS on the gut microbiota and important organs such as the liver and intestine were determined.

Based on the results of hematoxylin and eosin staining, it was revealed that there was a considerable increase in the number of fatty drops in the liver of mice treated with DADS, similar to the liver of mice fed with a HFD. The result of the 
oil-red $\mathrm{O}$ staining confirmed this result, and fat deposition in the liver was more notable in the mice treated with a low-dose of DADS compared with mice treated with a high-dose of DADS. In addition, DADS increased the body weight of mice fed with HFD. These results were unexpected, as previous studies have demonstrated that components from garlic had anti-obesity, anti-diabetic, hypolipidemic properties, and cardioprotective and hepatoprotective functions (25-29). Serum NEFA, TC, HDL-C and LDL-C were increased in the NFD group treated with a low-dose of DADS, and in the HFD groups. High levels of LDL has long been recognized as an important factor for abnormal lipid metabolism. Shi et al (30) reported that HDL-C levels were also significantly associated with atherosclerosis resulting from HFD. Changes in serum TC, LDL-C and HDL-C in the present study corroborated the results of Shi et al (30), suggesting that the functions of HDL-C require further study.

Based on the results of the serum tests and histochemical staining, the expression pattern of lipid-metabolism associated genes in the liver was measured using RT-qPCR and western blotting. The results demonstrated that the expression of hepatic lipase, HNF4 $\alpha$, FXR, Abcg5, ACC $\alpha 1$, DGAT1, SREBP-2 and PPAR $\gamma$ were significantly decreased in the HFD groups and in the NFD group treated with a low-dose of DADS, whereas the expression of CD36 was increased in the HFD group treated with a high-dose of DADS, as well as in the NFD group treated with a low-dose of DADS. The expression of Fasn, SREBP-1 and HMGCoAR were decreased in the HFD groups and in the NFD groups treated with low or high-doses of DADS compared with the NFD control group. In the NFD groups, the expression of Lipin 1 was significantly decreased in the group treated with a low-dose of DADS compared with the control and the high-dose groups. Lipin-1 is a mammalian phosphatidic acid phosphatase (PAP), which is required for lipid synthesis through its function as a PAP enzyme $(31,32)$. Lipin1 also functions as a transcriptional coactivator of lipid metabolism genes, which forms a physical complex with PGC-1 $\alpha$ and PPAR $\alpha$ to control the expression of genes involved in fatty acid oxidation and mitochondrial metabolism (32). Lipase is an enzyme that breaks down triglycerides into free fatty acids and glycerol, and it has previously been reported that lower hepatic lipase activity may be associated with higher concentrations of LDL triglycerides, thus increasing cardiovascular risk (33). The results of the present study revealed that a low dose of DADS decreased the expression levels of hepatic lipase in the liver of mice, promoting the formation of fatty liver. HNF4 $\alpha$ and FXR serve key roles in the regulation of bile acids (BA) (34), and the results of the present study revealed that a low-dose of DADS could decrease the expression of $\mathrm{HNF} 4 \alpha$ and FXR in the liver of mice. As HNF4 $\alpha$ regulates the expression of CYP7A1 directly, a significant decrease of $\mathrm{HNF} 4 \alpha$ may decrease the production of BA, which worsened fat deposition in the liver. Fatty acid translocase CD36 (FAT/CD36) regulates the uptake and intracellular transport of long-chain fatty acids in different cell types, and it has been reported that the upregulation of hepatic CD36 is significantly associated with IR and increased steatosis in patients with fatty liver (35), consistent with the results of the present study. DGAT1, DGAT2, SREBP-1, SREBP-2, HMGcoAR serve important roles in lipid synthesis (36-39), and the results of the present study demonstrated that DADS downregulated the expression of these genes. Another important gene in lipogenesis is stearoyl-CoA desaturase-1 (SCD1), which catalyzes the rate-limiting reaction of the synthesis of monounsaturated fatty acids, and it has been reported that elevated SCD1 activity has been implicated in a number of disorders, including obesity, diabetes and atherosclerosis (40). de Fourmestraux et al (41) indicated that increased expression of Scd-1 and the other lipogenic liver genes may be a critical step in the development of obesity when mice were fed with a HFD. The results of the present study revealed that the expression of SCD1 protein was significantly increased in the NFD groups treated with low and high-doses of DADS, suggesting that SCD1 may serve an important role in the development of fatty liver caused by DADS. Fibroblast growth factor (FGF)-21 and FGF19 (in mice, FGF15) exhibit opposite changes in expression levels in obesity $(42,43)$, and the results of the present study revealed that the expression of FGF15 was decreased, whereas expression of FGF21 was increased in the liver of mice treated with a low-dose of DADS (although the change was not significant), indicating that a low-dose of DADS may induce fat deposition in the liver.

Recently, focus has been placed on the gut microbiota after it was demonstrated that alterations in the gut microbiota were associated with a wide range of diseases, including obesity, diabetes, cardiovascular diseases and tumors (19,44-46). In the present study, the effects of DADS on gut microbiota were determined by performing a $16 \mathrm{~S}$ rDNA gene analysis, and the results revealed that the NFD group treated with a low-dose of DADS decreased Bacteroidetes and increased Firmicutes compared with the control and high-dose groups at the phyla taxonomic level. Turnbaugh et al (47) reported that obesity was associated with changes in the relative composition of the two dominant bacterial divisions, Bacteroidetes and Firmicutes, by comparing the gut microbiota of genetically obese mice and their lean littermates, as well as those of obese and lean human volunteers. It was revealed that the obese individuals had higher levels of Firmicutes and lower levels of Bacteroidetes (47). Islam et al (48) fed rats supplemented with different concentrations of cholic acid, and revealed that rats demonstrated increased Firmicutes levels accompanied by decreased Bacteroidetes levels, similar to that of the obesity-associated gut microbiome induced by high-fat diets. Recently, Zheng et al (49) evaluated the effects of dietary fat-induced BA changes on the shaping of the gut microbial composition in mice, and revealed that a HFD could induce the secretion of BA and the concentration and species of BA in intestines increased rapidly, which resulted in an obesity-type gut microbiota. The results of the present study demonstrated that a low-dose of DADS decreased Bacteroidetes levels and increased Firmicutes levels, similar to the gut microbiota composition of the HFD mice, and these results confirmed the phenotype of the mice treated with a low-dose of DADS that developed fatty liver. The results of the RT-qPCR in the present study demonstrated that the expression of HNF4 $\alpha$ and FXR was decreased in the liver of the mice treated with a low-dose of DADS, and thus, it was concluded that a low-dose of DADS may alter the ratio of Firmicutes to Bacteroidetes by regulating the expression of BA secretion and metabolism-associated genes, which was in 
agreement with the results of the study by Zheng et al (49), as they demonstrated that the changes in the concentration and species of BA in the intestine occurred earlier than the change in the gut microbiota.

Gut microbiota provide the enzymatic and metabolic pathways involved in food digestion, xenobiotic metabolism and production of molecules such as amino acids, short-chain fatty acids and metabolites, which are essential for glycolysis, the tricarboxylic acid cycle, oxidative phosphorylation, and amino acid and fatty acid metabolism (50). In the present study, HFD upregulated the pathways of 'Transporters', 'ABC transporters', 'DNA repair and recombination proteins', 'Ribosome', 'Purine metabolism', 'Peptidases', 'Pyrimidine metabolism', 'Two-component system', 'Chromosome', 'Amino acid related enzymes', 'Transcription factors', 'Amino sugar and nucleotide sugar metabolism', 'Methane metabolism', 'Ribosome Biogenesis', 'Arginine and proline metabolism', 'DNA replication proteins', 'Aminoacyl-tRNA biosynthesis', 'Carbon fixation pathways in prokaryotes', 'Alanine, aspartate and glutamate metabolism'. Similarly, the top 20 pathways of the NFD group treated with a low-dose of DADS were also upregulated compared with the NFD control group and the NFD group treated with a high-dose of DADS, indicating that a low-dose of DADS may affect the gut microbiota and impact the pathways involved in protein and amino acid metabolism and synthesis, lipid transport, oxidative phosphorylation and carbon metabolism. The effects of a low-dose of DADS on the structure of gut microbiota and pathways were similar to a HFD. It has been reported that colonocyte metabolism is directed towards oxidative phosphorylation during homeostasis of microbiota, maintaining a gut flora environment dominated by obligate anaerobic bacteria (51). Alterations of the metabolism of the colonic epithelium may increase epithelial oxygenation, resulting in an expansion of facultative anaerobic bacteria, which is a marker for dysbiosis in the colon (51). The results of the present study revealed that the oxidative phosphorylation pathway was significantly upregulated in the NFD group treated with a low-dose of DADS compared with the NFD control and NFD groups treated with a high-dose of DADS, but was significantly downregulated in the HFD group treated with a low-dose of DADS compared with the HFD control and the HFD groups treated with a high-dose of DADS. Therefore, the effects of DADS on metabolism and gut microbiota depend on dose and fat content of the food, and the underlying molecular mechanisms require further study.

In the present study, the phenotypes of mice fed with low and high-doses of DADS had similarities and differences. In the HFD groups, the body weight of mice treated with low and high doses of DADS was higher than the other groups. The levels of TC and TG were higher in the liver of mice treated with a low-dose of DADS in both the NFD and HFD groups. The results of the hematoxylin and eosin and oil-red $\mathrm{O}$ staining demonstrated that both low and high doses of DADS resulted in fat deposition in the liver of mice in the NFD and HFD groups, but the NFD group treated with a low-dose of DADS exhibited more severe instances of fatty liver. According to the results of the RT-qPCR, the expression patterns of the majority of the lipid metabolism-associated genes in the liver of the NFD mice treated with a low-dose of DADS was similar to that of the HFD groups. A low-dose of DADS had similar effects on lipid metabolism to a HFD and a larger effect than a high-dose of DADS.

Taken together, the results of the present study identified a notable phenomenon in mice fed with a normal diet. Treatment with DADS resulted in fatty liver development and an increased ratio of Firmicutes to Bacteroidetes in gut microbiota, similar to the phenotypes of mice fed with HFD. In addition, DADS could increase the body weight of mice fed with HFD. It was concluded that DADS may exert its effects on lipid metabolism and gut microbiota through regulation of the expression of genes associated with lipogenesis and lipid metabolism.

\section{Acknowledgements}

The authors would like to thank Dr Shenghua Piao from Guangdong Metabolic Disease Research Center of Integrated Chinese and Western Medicine, Guangdong Pharmaceutical University (Guangzhou, China) for helping with the language in the article.

\section{Funding}

This study was supported by the National Natural Science Foundation of China (grant. nos. 81803912 and 31671520), the Science and Technology Project of Guangdong Province (grant. nos. 2016B050501003, and 2017B050504005), the Scientific Research Project of the Administration of Traditional Chinese Medicine of Guangdong Province (grant. no. 20182079), the Characteristic Innovation Project (Natural Science) of the Education Department of Guangdong Province and the 'Innovation Strong School Project' of Guangdong Pharmaceutical University (grant. no. 2017KTSCX102) and the Science and Technology Project of Yue-Xiu District of Guangzhou (grant. no. 2018-WS-011).

\section{Availability of data and materials}

The datasets generated and analyzed during the present study are available from the corresponding author upon reasonable request.

\section{Authors' contributions}

ZL and JG and YY designed the study and conceived the report. YY analyzed and interpretated the results, wrote the first draft of the manuscript and revised it critically. FY, HW, MH, SLi, QW, CY, XZ, SLiu, YLei, LY, GC and YLiu performed the experiments. SLiu and YLei created the figures. All authors read and approved the final manuscript.

\section{Ethics approval and consent to participate}

The animal experiments were approved by The Committee on Laboratory Animal Care and Use of Guangdong Pharmaceutical University (Guangzhou, China).

\section{Patient consent for publication}

Not applicable. 


\section{Competing interests}

The authors declare that they have no competing interests.

\section{References}

1. Nam H, Jung H, Kim Y, Kim B, Kim KH, Park SJ and Suh JG: Aged black garlic extract regulates lipid metabolism by inhibiting lipogenesis and promoting lipolysis in mature 3T3-L1 adipocytes. Food Sci Biotechnol 27: 575-579, 2017.

2. Li Z, Le W and Cui Z: A novel therapeutic anticancer property of raw garlic extract via injection but not ingestion. Cell Death Discov 4: 108, 2018

3. Dwivedi VP, Bhattacharya D, Singh M, Bhaskar A, Kumar S, Fatima S, Sobia P, Kaer LV and Das G: Allicin enhances antimicrobial activity of macrophages during Mycobacterium tuberculosis infection. J Ethnopharmacol 243: 111634, 2019.

4. Behrouj H, Ziamajidi N, Abbasalipourkabir R, Goodarzi MT and Saidijam M: Hypoglycemic and antioxidant effects of oral administration of garlic extract in the livers of type 1 diabetic rats. J Basic Clin Physiol Pharmacol 30: 245-250, 2018.

5. Das B and Sinha D: Diallyl disulphide suppresses the cannonical Wnt signaling pathway and reverses the fibronectin-induced epithelial mesenchymal transition of A549 lung cancer cells. Food Funct 10: 191-202, 2019.

6. Su B, Su J, Zeng Y, Ding E, Liu F, Tan T, Xia H, Wu YH Zeng X, Ling H, et al: Diallyl disulfide inhibits TGF- $\beta 1$-induced upregulation of Rac1 and $\beta$-catenin in epithelial-mesenchymal transition and tumor growth of gastric cancer. Oncol Rep 39: 2797-2806, 2018

7. Li Y, Wang Z, Li J and Sang X: Diallyl disulfide suppresses FOXM1-mediated proliferation and invasion in osteosarcoma by upregulating miR-134. J Cell Biochem: Nov 1, 2018 (Epub ahead of print).

8. Ko JW, Jeong SH, Kwon HJ, Shin NR, Seo YS, Kim JC, Shin IS and Kim JS: Preventive effect of garlic oil and its organosulfur component diallyl-disulfide on cigarette smoke-induced airway inflammation in mice. Nutrients 10: E1659, 2018.

9. Singh AK, Bishayee A and Pandey AK: Targeting histone deacetylases with natural and synthetic agents: An emerging anticancer strategy. Nutrients 10: E731, 2018.

10. Yang Y, Chi Z, Gao R and Lei Z: The roles of natural compounds in epigenetics. Nat Product Commun 13: 1067-1072, 2018.

11. Schroeder BO and Bäckhed F: Signals from the gut microbiota to distant organs in physiology and disease. Nat. Med 22: 1079-1089, 2016

12. Gart E, Souto Lima E, Schuren F, de Ruiter CGF, Attema J, Verschuren L, Keijer J, Salic K, Morrison MC and Kleemann R: Diet-independent correlations between bacteria and dysfunction of gut, adipose tissue, and liver: A comprehensive microbiota analysis in feces and mucosa of the ileum and colon in obese mice with NAFLD. Int J Mol Sci 20: E1, 2018.

13. Hrncir T, Hrncirova L, Kverka $\mathrm{M}$ and Tlaskalova-Hogenova $\mathrm{H}$ : The role of gut microbiota in intestinal and liver diseases. Lab Anim 53: 271-280, 2018.

14. Filocamo A, Nueno-Palop C, Bisignano C, Mandalari G and Narbad A: Effect of garlic powder on the growth of commensal bacteria from the gastrointestinal tract. Phytomedicine 19 707-711, 2012

15. Wang Y, Guan M, Zhao X and Li X: Effects of garlic polysaccharide on alcoholic liver fibrosis and intestinal microflora in mice. Pharm Biol 56: 325-332, 2018

16. Nair AB and Jacob S: A simple practice guide for dose conversion between animals and human. J Basic Clin Pharm 7: 27-31, 2016.

17. Lai YS, Chen WC, Ho CT, Lu KH, Lin SH, Tseng HC, Lin SY and Sheen LY: Garlic essential oil protects against obesity-triggered nonalcoholic fatty liver disease through modulation of lipid metabolism and oxidative stress. J Agric Food Chem 62: 5897-5906, 2014

18. Yang YH, Lei ZL, Huang L, Yang F, Zhang N, Yuan J, Li K, Chen $\mathrm{J}$ and Zhang J: Antitumor ability of berberine accompanied by modulation of gut microbiome in sarcoma-180 tumor-bearing mice. Int J Pharmacol 14: 460-470, 2018.

19. Caporaso JG, Kuczynski J, Stombaugh J, Bittinger K, Bushman FD, Costello EK, Fierer N, Peña AG, Goodrich JK, Gordon JI, et al: QIIME allows analysis of high-throughput community sequencing data. Nat Methods 7: 335-336, 2010.
20. Edgar RC: UPARSE: Highly accurate OTU sequences from microbial amplicon reads. Nat Methods 10: 996-998, 2013.

21. Wang Q, Garrity GM, Tiedje JM and Cole JR: Naive Bayesian classifier for rapid assignment of rRNA sequences into the new bacterial taxonomy. Appl Environ Microbiol 73: 5261-5267, 2007.

22. Kemp PF and Aller JY: Bacterial diversity in aquatic and other environments: What 16S rDNA libraries can tell us. FEMS Microbiol Ecol 47: 161-177, 2004.

23. Frosini BV: Descriptive measures of ecological diversity. In: Environmetrics, in Encyclopedia of Life Support Systems (EOLSS), Developed under the Auspices of the UNESCO. Eolss Publishers, Oxford, 2004. http://www.eolss.net/ebooks/ sample\%20chapters/c02/e4-26-01-01.pdf.

24. Segata N, Izard J, Waldron L, Gevers D, Miropolsky L, Garrett WS and Huttenhower C: Metagenomic biomarker discovery and explanation. Genome Biol 12: R60, 2011.

25. Bae J, Kumazoe M, Fujimura Y and Tachibana H: Diallyl disulfide potentiates anti-obesity effect of green tea in high-fat/high-sucrose diet-induced obesity. J Nutr Biochem 64: 152-161, 2019.

26. Ryu JH and Kang D: Physicochemical properties, biological activity, health benefits, and general limitations of aged black garlic: A review. Molecules 22: E919, 2017.

27. Hosseini A and Hosseinzadeh $\mathrm{H}$ : A review on the effects of Allium sativum (Garlic) in metabolic syndrome. J Endocrinol Invest 38: 1147-1157, 2015.

28. Elkayam A, Mirelman D, Peleg E, Wilchek M, Miron T, Rabinkov A, Oron-Herman M and Rosenthal T: The effects of allicin on weight in fructose-induced hyperinsulinemic, hyperlipidemic, hypertensive rats. Am J Hypertens 16: 1053-1056.

29. Lee CG, Rhee DK, Kim BO, Um SH and Pyo S: Allicin induces beige-like adipocytes via KLF15 signal cascade. J Nutr Biochem 64: 13-24, 2019.

30. Shi Q, Hornsby PJ, Meng Q, Vandeberg JF and Vandeberg JL: Longitudinal analysis of short-term high-fat diet on endothelial senescence in baboons. Am J Cardiovasc Dis 3: 107-119, 2013.

31. You M, Jogasuria A, Lee K, Wu J, Zhang Y, Lee YK and Sadana P: Signal transduction mechanisms of alcoholic fatty liver disease: Emerging role of Lipin-1. Curr Mol Pharmacol 10: 226-236, 2017

32. Chen Y, Rui BB, Tang LY and Hu CM: Lipin family proteins-key regulators in lipid metabolism. Ann Nutr Metab 66: 10-18, 2015.

33. Silbernagel G, Scharnagl H, Kleber ME, Delgado G, Stojakovic T, Laaksonen R, Erdmann J, Rankinen T, Bouchard C, Landmesser U, et al: LDL triglycerides, hepatic lipase activity, and coronary artery disease: An epidemiologic and Mendelian randomization study. Atherosclerosis 282: 37-44, 2019.

34. Wang Y, Matye D, Nguyen N, Zhang Y and Li T: HNF4 $\alpha$ regulates CSAD to couple hepatic taurine production to bile acid synthesis in mice. Gene Expr 18: 187-196, 2018.

35. Miquilena-Colina ME, Lima-Cabello E, Sánchez-Campos S, García-Mediavilla MV, Fernández-Bermejo M, LozanoRodríguez T, Vargas-Castrillón J, Buqué X, Ochoa B, Aspichueta P, et al: Hepatic fatty acid translocase CD36 upregulation is associated with insulin resistance, hyperinsulinaemia and increased steatosis in non-alcoholic steatohepatitis and chronic hepatitis C. Gut 60: 1394-1402, 2011

36. Seo YJ, Lee K, Song JH, Chei S and Lee BY: Ishige okamurae extract suppresses obesity and hepatic steatosis in high fat diet-induced obese mice. Nutrients 10: E1802, 2018

37. Bozic M, Guzmán C, Benet $\mathrm{M}$, Sánchez-Campos $\mathrm{S}$, García-Monzón C, Gari E, Gatius S, Valdivielso JM and Jover R: Hepatocyte vitamin D receptor regulates lipid metabolism and mediates experimental diet-induced steatosis. J Hepatol 65: 748-757, 2016.

38. Zhang F, Sun W, Chen J, Jiang L, Yang P, Huang Y, Gong A, Liu S and Ma S: SREBP-2, a new target of metformin? Drug Des Devel Ther 12: 4163-4170, 2018.

39. Sakr HF, Hussein AM, Eid EA and AlKhateeb M: Possible mechanisms underlying fatty liver in a rat model of male hypogonadism: A protective role for testosterone. Steroids 135: 21-30, 2018.

40. Liu J, Cinar R, Xiong K, Godlewski G, Jourdan T, Lin Y, Ntambi JM and Kunos G: Monounsaturated fatty acids generated via stearoyl CoA desaturase- 1 are endogenous inhibitors of fatty acid amide hydrolase. Proc Natl Acad Sci USA 110: 18832-188327, 2013.

41. de Fourmestraux V, Neubauer H, Poussin C, Farmer P, Falquet L, Burcelin R, Delorenzi M and Thorens B: Transcript profiling suggests that differential metabolic adaptation of mice to a high fat diet is associated with changes in liver to muscle lipid fluxes. J Biol Chem 279: 50743-50753, 2004. 
42. Gallego-Escuredo JM, Gómez-Ambrosi J, Catalan V, Domingo P, Giralt M, Frühbeck G and Villarroya F: Opposite alterations in FGF21 and FGF19 levels and disturbed expression of the receptor machinery for endocrine FGFs in obese patients. Int J Obes (Lond) 39: 121-129, 2015

43. Zhang F, Yu L, Lin X, Cheng P, He L, Li X, Lu X, Tan Y, Yang H, Cai $\mathrm{L}$ and Zhang C: Minireview: Roles of fibroblast growth factors 19 and 21 in metabolic regulation and chronic diseases. Mol Endocrinol 29: 1400-1413, 2015.

44. Gérard $C$ and Vidal H: Impact of gut microbiota on host glycemic control. Front Endocrinol (Lausanne) 10: 29, 2019.

45. Halawa MR, El-Salam MA, Mostafa BM and Sallout SS: The gut microbiome, lactobacillus acidophilus; relation with type 2 diabetes mellitus. Curr Diabetes Rev: Feb 6, 2019 (Epub ahead of print).

46. Ai D, Pan H, Han R, Li X, Liu G and Xia LC: Using decision tree aggregation with random forest model to identify gut microbes associated with colorectal cancer. Genes (Basel) 10: E112, 2019.

47. Turnbaugh PJ, Ley RE, Mahowald MA, Magrini V, Mardis ER and Gordon JI: An obesity-associated gut microbiome with increased capacity for energy harvest. Nature 444: 1027-1031, 2006.
48. Islam KB, Fukiya S, Hagio M, Fujii N, Ishizuka S, Ooka T, Ogura Y, Hayashi T and Yokota A: Bile acid is a host factor that regulates the composition of the cecal microbiota in rats. Gastroenterology 141: 1773-1781, 2011.

49. Zheng X, Huang F, Zhao A, Lei S, Zhang Y, Xie G, Chen T, Qu C, Rajani C, Dong B, et al: Bile acid is a significant host factor shaping the gut microbiome of diet-induced obese mice. BMC Biol 15: 120, 2017.

50. Belizário JE, Faintuch J and Garay-Malpartida M: Gut microbiome dysbiosis and immunometabolism: New frontiers for treatment of metabolic diseases. Mediators Inflamm 2018: 2037838, 2018.

51. Litvak Y, Byndloss MX and Bäumler AJ: Colonocyte metabolism shapes the gut microbiota. Science 362: eaat9076, 2018.

(i) $\Theta$ This work is licensed under a Creative Commons Attribution-NonCommercial-NoDerivatives 4.0 International (CC BY-NC-ND 4.0) License. 